Archives de sciences sociales des religions

160 | octobre-décembre 2012

Bulletin Bibliographique

\title{
Enquête chez les biblistes
}

Gérard Leclerc

\section{(2) OpenEdition}

Journals

Édition électronique

URL : http://journals.openedition.org/assr/24562

DOI : 10.4000/assr.24562

ISSN : $1777-5825$

Éditeur

Éditions de l'EHESS

Édition imprimée

Date de publication : 30 décembre 2012

Pagination : 75-89

ISSN : 0335-5985

Référence électronique

Gérard Leclerc, «Enquête chez les biblistes », Archives de sciences sociales des religions [En ligne],

160 | octobre-décembre 2012, mis en ligne le 30 décembre 2012, consulté le 21 avril 2019. URL http://journals.openedition.org/assr/24562 ; DOI : 10.4000/assr.24562

(C) Archives de sciences sociales des religions 


\section{Savoir bibliste}

À propos de :

LASSAVE Pierre, L'Appel du texte. Sociologie du savoir bibliste, Rennes, Presses universitaires de Rennes, coll. "Sciences des religions", 2011, 210 p.

\section{Gérard Leclerc}

\section{Enquête chez les biblistes}

Ce dernier ouvrage de Pierre Lassave est une enquête sociologique sur la profession de "bibliste ", laquelle présente des caractéristiques atypiques à plusieurs égards. Si l'on s'accorde généralement à situer le métier de « bibliste " à l'intérieur du monde des chercheurs scientifiques, les nomenclatures ne lui reconnaissent guère en général une visibilité propre à l'intérieur des disciplines historiques et textuelles. L'ouvrage rappelle que le "savoir bibliste " ne s'est constitué que tout récemment dans sa spécificité. Pour l'essentiel, il fait partie du champ des « sciences religieuses » apparu dans le cadre de la sécularisation théologicopolitique et culturelle. En Allemagne, le processus était engagé depuis le début du XIX siècle. En France, cette étape est jalonnée par les noms emblématiques de Renan (Vie de Jésus, 1863) et de Loisy (L'Évangile et l'Église, 1902). La séparation de l'Église et de l'État en 1905 est indissociable de l'institutionnalisation de l'exégèse biblique dans le monde universitaire français, fondée sur une lecture de type purement historique (philologique, ethnographique, sociologique, etc.). L'un des effets des débats apparus à cette occasion aura été la naissance officielle d'une nouvelle discipline universitaire, avec les "sciences religieuses ", qui font leur apparition en 1886 dans le cadre de l'École pratique des hautes études (EPHE). Cette naissance d'une lecture purement "laïque» de la Bible dans le monde universitaire marque la fin du monopole de l'Église sur la lecture légitime de la Bible. Mais la lecture confessionnelle garde sa place propre à l'intérieur même du monde de la recherche et marque ses positions, à travers la création de l'École pratique des études bibliques de Jérusalem (1890).

L'auteur rappelle les deux domaines principaux qui furent porteurs de controverses entre la lecture traditionnelle et les lectures nouvelles. Pour ce qui concerne l'Ancien Testament, il s'agit essentiellement de la "question du Pentateuque ", c'est-à-dire les problèmes liés à la mise en évidence, au sein des premiers livres de la Bible, de plusieurs sources d'époque différente et ayant des visées littéraires 
distinctes. L'hypothèse "documentaire » acquiert à la fin du XIX siècle sa respectabilité au sein de l'exégèse historico-critique allemande avec les travaux de Wellhausen. Concernant le Nouveau Testament, la question porte en particulier sur la légitimité et la possibilité d'avoir accès, par-delà la Tradition théologique, à un "Jésus historique " distinct du « Jésus de la foi ». Loisy et le P. Lagrange, qui se situent tous deux à l'intérieur de l'orthodoxie catholique, se font en France les avocats de cette approche, ce qui leur vaudra bien des déboires dans leurs rapports avec le Magistère. L'auteur expose les étapes marquantes de cette histoire propre à l'exégèse biblique française, avant d'étudier les effets de l'institutionnalisation de la profession d'exégète biblique au sein du monde universitaire français.

Le chapitre II retrace l'essor de la recherche bibliste dans le monde francophone actuel. À cet égard, il fait remarquer que son étude porte sur un ensemble culturel bien délimité. L'école biblique française (ou francophone) est loin d'être le courant principal de ce domaine de recherches. L'auteur rappelle que l'exégèse a parlé et écrit essentiellement en allemand jusqu'à une date toute récente, mais qu'elle est maintenant principalement de langue anglaise (où jouent un rôle dominant les universités américaines). Pour ce qui concerne la France, l'auteur note le développement récent de nombreux cercles et réseaux biblistes; le rôle des Éditions du Cerf, celui de l'ACFEB (Association catholique française pour d'étude de la Bible), de la BOSEB (Bibliothèque œcuménique et scientifique pour l'étude de la Bible), du RRENAB (Réseau de recherches en analyse narrative des textes bibliques), etc.

Mais l'enquête est orientée par un questionnement précis : quelle est la spécificité du métier de "bibliste " ? De quel type de "savoir » relève le discours bibliste ? En quel sens s'agit-il d'une discipline universitaire et, plus précisément, d'un discours scientifique ? Pour répondre à cette question, l'auteur s'est livré à une observation ethnographique de terrain en fréquentant plusieurs colloques qui se sont déroulés entre 2006 et 2009 dans des grandes villes universitaires (Paris, Lyon), qui sont aussi des capitales religieuses. Il a en outre procédé à des entretiens avec des chercheurs. Y sont étudiées les diverses motivations d'entrée dans la carrière de bibliste. Plusieurs exemples de carrières récentes sont proposés sous forme de portraits, à travers un usage original de la notion sociologique de «carrière ", élaborée par la sociologie des professions de Everett Hughes. L'approche ethnographique est l'occasion de descriptions minutieuses et fines du travail de bibliste. Appartenant lui-même au monde universitaire, l'auteur note "l'extériorité relative " qui est la sienne " par rapport au microcosme rencontré ". Il est ainsi amené à noter la spécificité du " monde bibliste », un monde qui jouit d'une "certaine autonomie épistémique ", mais qui souffre aussi d'une certaine "fragilité institutionnelle ", dans la mesure où il se situe au carrefour de l'autorité de l'Église et de celle de l'Université.

Les biblistes français sont les héritiers de la situation historique qui a vu la séparation de l'Église et de l'État, et la naissance d'une lecture professionnelle 
« laïque » de la Bible, dans le cadre de l'université (la plupart sont des enseignantschercheurs). Mais en même temps, ils sont, dans leur très grande majorité, des croyants (principalement chrétiens, de diverses obédiences catholiques et protestantes) assumant leur foi, donc une lecture "confessante ". Ils se réfèrent ainsi à deux catégories de normes et d'institutions : normes scientifiques/normes religieuses ; Institution universitaire/Église. Deux approches de la Bible sont ainsi mises en tension : la Bible comme Texte sacré, comme Parole de Dieu, et la Bible comme Texte canonique, littéraire, historique, narratif, etc. Le titre de l'ouvrage semble faire référence à cette double approche : l' " appel du texte " paraît renvoyer à la fois à l'analyse historique et littéraire et à un " appel » de type différent, qui évoque la "vocation ", dont la dimension " extra-professionnelle » semble présente en filigrane. L'auteur montre d'ailleurs comment la carrière de chercheur bibliste a pu se greffer sur certaines formes de "vocation religieuse ».

Même si les nouvelles méthodes exégétiques sont le témoignage d'une certaine " sécularisation du métier de bibliste ", l'auteur cherche à " appréhender la nature objective d'un savoir que l'on a peine à discerner comme discipline universitaire ". L'auteur est ainsi amené à s'interroger sur les "incertitudes de la compétence bibliste ». On peut se demander si le travail de bibliste - un «savoir qui fait métier sans devenir profession »- ne pourra jamais constituer une " profession scientifique " autonome. Le statut de la discipline «bibliste » ne repose pas sur une méthode spécifique (ses méthodes sont essentiellement celles de l'analyse historique et textuelle : linguistique, philologique, herméneutique, etc.), mais sur son objet singulier : un Texte canonique, qui est en outre, pour la grande majorité des chercheurs, un Texte sacré incarnant la Parole de Dieu. En tant que discipline et en tant que savoir, l'exégèse biblique revendique donc un statut épistémique à la fois ordinaire/normal/universitaire et extraordinaire/exceptionnel. Comme s'il existait une sorte d'extraterritorialité de son objet qui le rendrait inassimilable par une approche purement comparative, vécue par beaucoup comme réductrice et, d'une certaine façon, comme illégitime.

La dernière partie de l'enquête porte sur la "vulgarisation ", ou mieux, sur la «médiatisation » des travaux des biblistes, c'est-à-dire sur leur réception dans le grand public. Elle fait en particulier référence à la diffusion par la chaîne culturelle Arte de trois séries documentaires et historiques (Corpus Christi en 1997-1998, L'Origine du christianisme en 2004, L'Apocalypse en 2008) qui présentaient les origines du christianisme telles qu'elles sont aujourd'hui reconstruites par un ensemble très large de chercheurs. L'analyse aborde la façon dont ces derniers ont réagi à la diffusion dans le grand public de certains aspects de leur contribution personnelle. Certains s'en réjouissent, estimant qu'elle contribue à augmenter la reconnaissance publique de leur discipline ; d'autres se préoccupent de la façon dont les cinéastes ont pu sélectionner et réinterpréter leurs travaux. Un autre mode de "médiatisation " des questions bibliques fut l'affaire du Da Vinci Code, d'abord sous la forme d'un roman de Dan Brown, 
puis sous celle d'un film hollywoodien. La dimension scandaleuse de l'affaire, pour l'Église et pour beaucoup de croyants tient à la façon dont ce roman pseudohistorique exploite l'espace de non-conformisme et de liberté ouvert par la critique biblique. On peut y voir à la fois l'apothéose et la damnation de la recherche bibliste, dans sa dimension de liberté et de distanciation par rapport à l'approche théologique et dogmatique traditionnelle.

En conclusion, l'ouvrage pose la question de la place de la recherche bibliste à l'intérieur des sciences humaines, en particulier les sciences philologiques et textuelles, dont le contenu semble se renouveler sous l'influence de l'approche narratologique. Également de leur place dans le champ qui pourrait se dessiner autour d'une approche comparative des grands Textes canoniques : Classiques chinois, Textes de la Révélation védique et hindoue, Textes bouddhistes, Coran et Hadith... Quels seraient les effets scientifiques, culturels, idéologiques et politiques d'une telle approche comparative, où l'exégèse biblique perdrait son droit d'exterritorialité, pour être mobilisée en tant que champ particulier d'une telle approche ? Les questions bibliques avaient été dominées au XIX et au XX ${ }^{\mathrm{e}}$ siècle par la question de la légitimation d'un nouveau mode de lecture - historique et philologique - de la Bible. L'originalité du travail de Pierre Lassave réside dans l'exercice d'un nouveau type de questionnement, caractérisé par une approche sociologique et ethnographique du monde des lecteurs professionnels de la Bible répondant à l'appel du Texte.

Gérard LECLERC

Université Paris VIII gerard.lec@wanadoo.fr

Jean Lambert

\section{L'Ancienne exégèse et la Nouvelle}

"Tout texte est le tissu de sa genèse " (Michel Serres)

Cette enquête sur des biblistes chrétiens se présente comme une sociologie paradoxale d'un savoir conséquent (et fort ancien), au carrefour de disciplines attestées, où se déploie un pluralisme irréductible de significations pour un objet partagé sinon unique. L'ethnographie de ce milieu composite est pionnière, et bienvenue en ces temps de regain d'intérêt pour le religieux. Elle interroge la nature de ce savoir à travers ses manuels (composition du Pentateuque, quête du Jésus historique : comment progresse l'histoire des mythes ?), ses institutions (les biblistes sont-ils des scientifiques ?), ses colloques (conflits d'interprétations, 